\title{
PP2A inhibitors suppress migration and growth of PANC-1 pancreatic cancer cells through inhibition on the Wnt/ $\beta$-catenin pathway by phosphorylation and degradation of $\boldsymbol{\beta}$-catenin
}

\author{
MENG-YAO WU ${ }^{1 *}$, XIN XIE $^{1,7^{*}}$, ZE-KUAN XU ${ }^{2}$, LI XIE $^{2}$, ZHENG CHEN $^{2}$, LIU-MEI SHOU $^{1}$, \\ FEI-RAN GONG ${ }^{3-5}$, YU-FENG XIE ${ }^{1}$, WEI LI ${ }^{1,2}$ and MIN TAO ${ }^{1,6}$ \\ ${ }^{1}$ Department of Oncology, The First Affiliated Hospital of Soochow University, Suzhou, Jiangsu 215006; \\ ${ }^{2}$ Department of General Surgery, The First Affiliated Hospital of Nanjing Medical University, Nanjing, Jiangsu 210029; \\ ${ }^{3}$ Department of Hematology, ${ }^{4}$ Jiangsu Institute of Hematology, and ${ }^{5}$ Key Laboratory of Thrombosis and Hemostasis \\ of Ministry of Health, The First Affiliated Hospital of Soochow University, Suzhou, Jiangsu 215006; \\ ${ }^{6}$ Jiangsu Institute of Clinical Immunology, Suzhou, Jiangsu 215006, P.R. China
}

Received March 9, 2014; Accepted May 27, 2014

DOI: $10.3892 /$ or.2014.3266

\begin{abstract}
Cantharidin is an active constituent of mylabris, a traditional Chinese medicine, and presents strong anticancer activity in various cell lines. Cantharidin is a potent and selective inhibitor of serine/threonine protein phosphatase $2 \mathrm{~A}$ (PP2A). Our previous studies revealed the prospect of application of cantharidin, as well as other PP2A inhibitors, in the treatment of pancreatic cancer. However, the mechanisms involved in the anticancer effect of PP2A inhibitors have not been fully explored. The Wnt/ $\beta$-catenin pathway is involved in cell migration and proliferation and participates in the progression of pancreatic cancer. If $\beta$-catenin is phosphorylated and degraded, the Wnt $/ \beta$-catenin pathway is blocked. PP2A dephosphorylates $\beta$-catenin and keeps the $\mathrm{Wnt} / \beta$-catenin pathway active. In the present study, we found that PP2A inhibitor treatment induced phosphorylation and degradation of $\beta$-catenin. The suppression on the migration and growth of PANC-1 pancreatic cancer cells could be attenuated by pretreatment with FH535, a $\beta$-catenin pathway inhibitor. Microarray showed that PP2A inhibitor treatment induced expression changes in 13 of 138 genes downstream of the $\beta$-catenin pathway. Real-time PCR further confirmed that FH535 attenuated the expression changes induced by PP2A inhibitors in 6 of these 13 candidate
\end{abstract}

Correspondence to: Professor Min Tao or Dr Wei Li, Department of Oncology, The First Affiliated Hospital of Soochow University, Suzhou, Jiangsu 215006, P.R.China

E-mail:mtao@medmail.com.cn

E-mail: liwei10@suda.edu.cn

Present address: ${ }^{7}$ Affiliated Hospital of Xuzhou Medical College, Xuzhou, Jiangsu, P.R. China

*Contributed equally

Key words: pancreatic cancer, Wnt/ $\beta$-catenin signal pathway, PP2A, phosphorylation genes. These 6 genes, VEGFB, Dkk3, KRT8, NRP1, Cacnalg and WISP2, have been confirmed to participate in the migration and/or growth regulation in previous studies. Thus, the phosphorylation- and degradation-mediated suppression on $\beta$-catenin participates in the cytotoxicity of PP2A inhibitors. Our findings may provide insight into the treatment of pancreatic cancer using a targeting PP2A strategy.

\section{Introduction}

Pancreatic cancer is one of the most fatal solid malignancies, with a 5-year survival rate of only $\sim 5 \%$. There is no method for early detection of the cancer and most patients with localized cancer have no recognizable symptoms. As a result, most patients are not diagnosed until the cancer metastasizes to other organs (1). Less than $20 \%$ of patients are eligible for curative resection and, of those, most experience recurrence of the cancer. Thus, an effective treatment and therapy are essential (2).

Mylabris is the dried body of the Chinese blister beetle. The use of mylabris as a traditional Chinese medicine in the treatment of tumor can be traced back to $>2,000$ years ago and it is still used as a folk medicine today (3). The active constituent of mylabris is cantharidin (3). In our previous studies, we found that cantharidin presented cytotoxicity through NF- $\kappa \mathrm{B}$ pathway-mediated apoptosis induction (4), JNK pathway-dependent growth inhibition $(3,5)$ and suppression on migration in breast cancer cells (6). It remains unclear whether cantharidin, as well as other PP2A inhibitors, could suppress the migration of pancreatic cancer cells. Moreover, the mechanism involved in the migration inhibition induced by cantharidin remains unknown.

The conserved Wnt/ $\beta$-catenin pathway regulates stem cell pluripotency and cell fate decisions during development $(7,8)$. Previous studies presented the participation of $\mathrm{Wnt} / \beta$-catenin pathway in cell migration and proliferation (9). In the absence of Wnt signal, $\beta$-catenin is phosphorylated by glycogen synthase kinase-3 $\beta$ (GSK-3 $\beta$ ) and then degraded by the ubiquitin-proteasome system. When Wnt ligands bind to frizzled 
receptors, GSK-3 $\beta$ activity is inhibited and unphosphorylated $\beta$-catenin accumulates in the cytoplasm and translocates into the nucleus, where it acts as a transcription cofactor with $\mathrm{T}$ cell factor/lymphoid enhancer factor TCF/LEF (9) and regulates the transcription of a variety of the target genes $(7,8)$. Dysregulation of Wnt/ $\beta$-catenin signaling and altered transcription of $\beta$-catenin/TCF-regulated genes are found in many cancers (10), including pancreatic cancer (11).

Mechanistically, cantharidin has been shown to be a potent and selective inhibitor of serine/threonine protein phosphatase 2A (PP2A) (3). Our previous studies revealed that cantharidin, as well as other PP2A inhibitors, fulfilled their anticancer effect through inhibition of PP2A and subsequent activation of several kinase pathways (3-6). A previous study proved that PP2A dephosphorylates $\beta$-catenin. Treatment of colon cancer cells with classical PP2A inhibitor, okadaic acid (OA), increases the phosphorylation of $\beta$-catenin (12).

Therefore, in the present study, we investigated whether cantharidin, as well as other PP2A inhibitors, could suppress the migration and growth of pancreatic cancer cells through phosphorylation and degradation of $\beta$-catenin-mediated inhibition on the $\mathrm{Wnt} / \beta$-catenin pathway.

\section{Materials and methods}

Cells and cultures. The human pancreatic cancer cell line PANC-1 was purchased from the American Type Culture Collection (ATCC; Manassas, VA, USA). Cells were maintained in DMEM medium (Gibco, Grand Island, NY, USA). Medium was supplemented with $10 \%$ fetal calf serum (Gibco), $100 \mathrm{U} / \mathrm{ml}$ penicillin and $100 \mathrm{mg} / \mathrm{ml}$ streptomycin at $37^{\circ} \mathrm{C}$ in a $5 \% \mathrm{CO}_{2}$ incubator with humidified atmosphere. Cells were passaged every 2-3 days for exponential growth.

Reagents. Cantharidin, OA and SP600125 were purchased from Enzo Life Sciences International (Plymouth Meeting, PA, USA). Norcantharidin (NCTD) was purchased from Sigma (St. Louis, MO, USA). FH535 was purchased from Millipore (Billerica, MA, USA).

Wound healing assay. The cells were seeded in 96-well plates at a density of $1 \times 10^{4}$ cells/well and grown to confluence. The monolayer culture was then artificially scrape-wounded with a sterile micropipette tip to create a denuded zone (gap) of constant width. Each well was washed with phosphate-buffered saline (PBS) twice to remove the detached cells before treatment. Cells that had migrated to the wounded region were observed using an XDS-1B inverted microscope (MIC Optical \& Electrical Instrument, Chongqing, China) and photographed (magnification, $\mathrm{x} 40$ ). Images were captured at various time points to monitor the wound healing process. The wound areas were measured using ImageJ (NHI, Bethesda, MA, USA).

MTT assay. Cellular growth was evaluated by the 3-[4,5-dimethylthiazol-2-yl]-2,5-diphenyl tetrazolium bromide (MTT) assay (13). The cells were seeded into 24-well tissue culture plates at $5 \times 10^{4}$ cells/well. After treatment, MTT (Sigma) was added to each well at a final concentration of $0.5 \mathrm{mg} / \mathrm{ml}$, followed by incubation at $37^{\circ} \mathrm{C}$ for $4 \mathrm{~h}$. The medium was then removed and $800 \mu 1$ dimethyl sulfoxide was added to each well. The absorbance of the mixture was measured at $490 \mathrm{~nm}$ using a microplate ELISA reader (Bio-Rad Laboratories, Hercules, CA, USA). The relative cell viability was calculated as follows: relative cell viability $=($ mean experimental absorbance $/$ mean control absorbance) x100\%.

Western blot analysis. Total protein was extracted using a lysis buffer containing $50 \mathrm{mM}$ Tris- $\mathrm{HCl}$ (pH 7.4), $150 \mathrm{mM} \mathrm{NaCl}$, $1 \%$ Triton X-100, 0.1\% SDS, 1 mM EDTA, supplemented with protease inhibitor cocktail kit (Roche, Indianapolis, IN, USA) and phosphatase inhibitor cocktail kit (Roche). The cytosol and nuclear extracts were prepared by NE-PER nuclear and cytoplasmic extraction reagents (Pierce Biotechnology, Rockford, IL, USA), supplemented with protease inhibitor cocktail kit (Roche). The protein extract was loaded, size-fractionated by SDS-polyacrylamide gel electrophoresis and transferred to PVDF membranes (Bio-Rad Laboratories). After blocking, the membranes were incubated with primary antibodies at $4^{\circ} \mathrm{C}$ overnight. Rabbit anti-phospho- $\beta$-catenin (Thr41/Ser45) antibody was purchased from Santa Cruz Biotechnologies (Santa Cruz, CA, USA). Mouse anti-active- $\beta$-catenin (unphosphorylated Ser37/Thr41, clone 8E7) antibody was purchased from Millipore. Mouse anti- $\beta$-catenin was purchased from Cell Signaling Technology (Beverly, MA, USA). Rabbit anti-RPL38 and rabbit anti-histone $\mathrm{H} 1$ antibodies were purchased from Proteintech Group (Chicago, IL, USA). The protein expression was determined using horseradish peroxidase-conjugated antibodies followed by enhanced chemiluminescence (ECL; Millipore) detection. The intensity of the bands was captured by JS-1035 image analysis scanning system (Peiqing Science \& Technology, Shanghai, China). RPL38 and histone H1 were used as internal controls for the total and nuclear extracts, respectively.

Microarray assay. Sample preparation and processing procedure were performed as described in detail in the Agilent GeneChip Expression Analysis Manual (Santa Clara, CA, USA). Differentially expressed genes were screened using Agilent 44K human whole-genome oligonucleotide microarrays. The selection criterion was defined as a $>1.5$-fold difference in the level of expression (difference in upregulated expression $>1.5$-fold and difference in downregulated expression $<0.67$-fold). Hierarchical clustering of samples was performed by average linkage algorithm using TIGR MultiExperiment Viewer (The Institute for Genomic Research, Rockville, MD, USA).

Real-time PCR. Total RNA was extracted using TRIzol reagent (Life Technologies) according to the manufacturer's protocol. After spectrophotometric quantification, $1 \mu \mathrm{g}$ total RNA was used for reverse transcription in a final volume of $20 \mu \mathrm{l}$ with AMV reverse transcriptase (Promega) according to the manufacturer's instructions. Aliquots cDNA of corresponding to equal amounts of RNA were used for the quantification of mRNA by real-time PCR using the LightCycler 96 real-time quantitative PCR detection system (Roche). The reaction system $(25 \mu \mathrm{l})$ contained the corresponding cDNA, forward and reverse primers and SYBR-Green PCR Master Mix (Roche). All data were analyzed using RPL38 gene expression as an internal standard. The specific primers are presented in Table I. 
Table I. Primers.

\begin{tabular}{lllr}
\hline Genes & \multicolumn{1}{c}{ Sense (5'-3') } & \multicolumn{1}{c}{ Antisense (5'-3') } & $\begin{array}{r}\text { Product } \\
\text { size (bp) }\end{array}$ \\
\hline VEGFB & GGACAGAGTTGGAAGAGGAGAC & GGGAGGAAGAGCCAGTTGTA & 130 \\
Dkk3 & GGGAGGAGATGGAAACAATG & ATGGAAAGAACTGCGTGGAA & 131 \\
MYC & CCTCCACTCGGAAGGACTATC & TTGTGTGTTCGCTCTTGAC & 142 \\
KRT8 & CAGGAGCTGATGAACGTCAA & TCATGTTCTGCATCCCAGAC & 106 \\
NRP1 & GACAGAAACTGGATGCCTGAA & CTTCCCACCCTGAATGATGA & 153 \\
Cacnalg & CACTCTCTGCCCAATGACAG & ACAAGACGGAGCCTGACTGA & 109 \\
WISP2 & ACTCCCTGCCTACACACACAG & TGCCTTCTCTTCATCCTACCC & 165 \\
PTGS2 & TAGGTGCATTGGAATCAAGC & GGAGAAACGAAGTGATGAGAAGA & 105 \\
BTRC & GCAGTCCAACCCAGATTAGTG & AAATGGCTCTCTTTCCGATACT & 135 \\
DAB2 & TGTGGCTTCTTCTCAACCTG & TTATTCCTCTGGATGGTCTGC & 104 \\
AHR & ACGAGGTCAAGAGATGGAGAC & TTCCCAGGTTCAGGCTATTC & 148 \\
TIAM1 & CTTCCCTCATCCCAGCAATA & CCTCCTCCTCCCAAGAGACT & 120 \\
UBXN8 & GCAGCAAAGAGCCAGAACTT & GGACATCGGAGAGCAACAGT & 143 \\
RPL38 & GCTGCTTGCTGTGAGTGTCT & AGATTTGGCATCCTTTCGTC & 149 \\
\hline
\end{tabular}

Statistical analysis. Each experiment was performed at least in triplicate. Results are expressed as the mean value \pm standard deviation (SD). Statistical analysis was performed using an unpaired Student's t-test. A P-value $<0.05$ was considered to indicate a statistically significant difference.

\section{Results}

Cantharidin suppresses cell migration through JNK pathwayindependent manner. Previously, we found that cantharidin suppressed the proliferation of pancreatic cancer cells $(3,5)$. However, whether cantharidin affects the migration of pancreatic cancer cells remains unknown. Thus, we investigated whether cantharidin suppresses the migration of pancreatic cancer PANC-1 cells using a wound healing assay. As shown in Fig. 1A, cantharidin suppressed the migration of PANC-1 cells in a time- and dose-dependent manner, suggesting the anticancer effect of cantharidin also involves the suppression on metastasis potential.

In our previous studies, cantharidin-induced activation of JNK suppressed pancreatic cancer cell growth $(3,5)$. As the JNK pathway is also involved in the migration regulation (14), we investigated whether cantharidin suppressed migration of PANC-1 cells through activation of the JNK pathway. Treatment with SP600125, the inhibitor of JNK, time-dependently suppressed migration of PANC-1 cells, suggesting the basal activity of the JNK pathway promoted migration of pancreatic cancer cells. Notably, pretreatment with SP600125 did not attenuate the cantharidin-induced suppression on migration, but even strengthened the cantharidin-induced suppression of cell migration (Fig. 1B), suggesting the activation of JNK impaired the migration suppression of cantharidin.

PP2A inhibitors suppress the $\beta$-catenin pathway through phosphorylation-mediated degradation of $\beta$-catenin.
Treatment with PP2A inhibitors, cantharidin, NCTD and OA, increased the phosphorylation level and decreased the nonphosphorylated form (active form) of $\beta$-catenin, accompanied by the reduction of total protein level of $\beta$-catenin (Fig. 2A). Blotting of nuclear protein proved the decreased nuclear distribution of $\beta$-catenin after treatment with PP2A inhibitors (Fig. 2B). These data suggested that PP2A inhibitors induced phosphorylation and degradation of $\beta$-catenin, which further resulted in the decreased nuclear translocation.

PP2A inhibitors suppress migration and growth of pancreatic cancer cells through suppression on $\beta$-catenin pathway. To investigate whether the suppression on the $\beta$-catenin pathway is involved in the anticancer effect of PP2A inhibitors, we used FH535, a classic Wnt/ $\beta$-catenin inhibitor (15), to verify whether blocking $\mathrm{Wnt} / \beta$-catenin could attenuate the migration and growth suppression caused by PP2A inhibitors. Treatment with FH535 suppressed the migration and growth of pancreatic cancer cells, suggesting $\beta$-catenin may participate in the progression of pancreatic cancer (Fig. 3A and B).

Cantharidin, NCTD and OA suppressed the migration and growth of pancreatic cancer cells, respectively. When cells were pretreated with FH535, the anti-migration and anti-growth effects of PP2A inhibitors were partially attenuated, suggesting that the anticancer effects of PP2A inhibitors were partially $\beta$-catenin pathway-dependent.

PP2A inhibitors induce expression changes of genes at the downstream of the $\beta$-catenin pathway. We performed microarray analyses to determine the mRNA expression changes of 138 genes downstream of $\beta$-catenin pathway (http://www.stanford.edu/group/nusselab/cgi-bin/wnt/target_genes) (Fig. 4A). Only those genes that significantly altered expression levels (by 1.5-fold) in all FH535, cantharidin and OA groups were chosen for further analysis. Of the 138 genes analyzed, 13 fulfilled this criterion (Fig. 4B). 
A
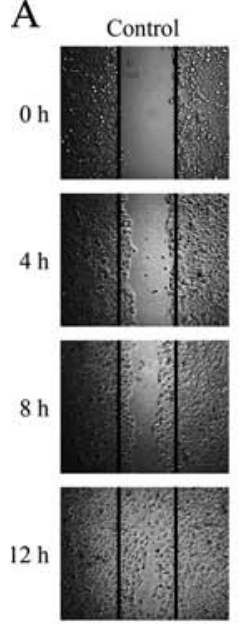

$\mathrm{CAN} 10 \mu \mathrm{M}$
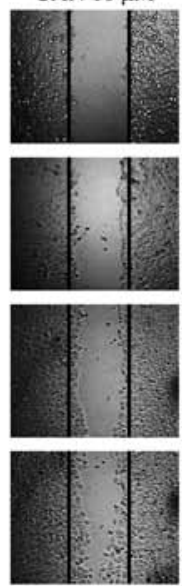

CAN $20 \mu \mathrm{M}$
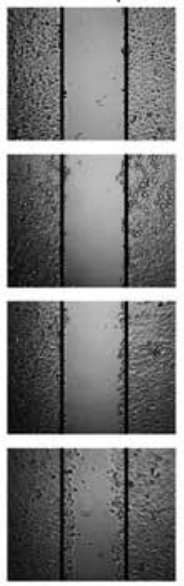

B
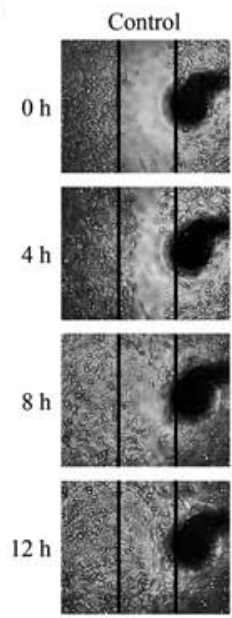
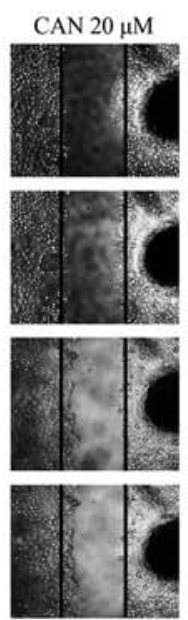
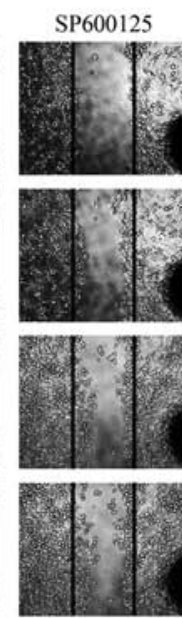
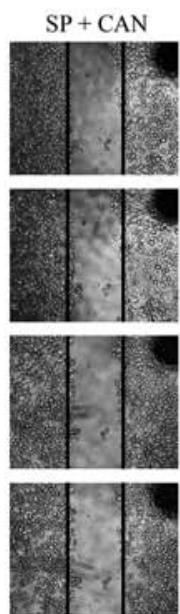
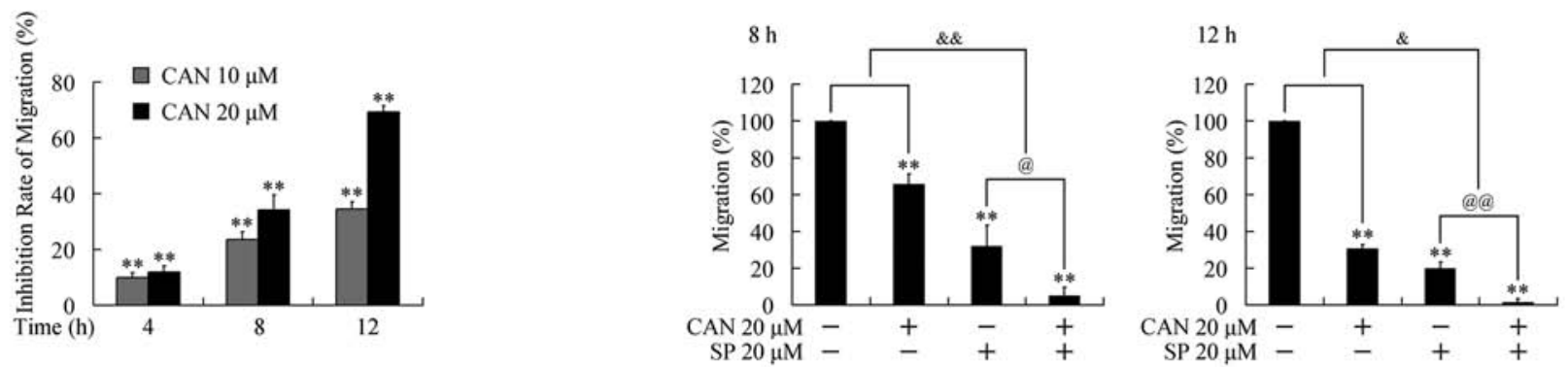

Figure 1. Cantharidin (CAN) suppresses PANC-1 cell migration. (A) CAN suppressed the migration of PANC-1 cells in a time- and dose-dependent manner. ${ }^{* *} \mathrm{P}<0.01$, significant differences from the control group. (B) Pretreatment with SP600125 (SP) strengthened the suppression on migration induced by CAN.

${ }^{* *} \mathrm{P}<0.01$, significant differences from the respective control groups. ${ }^{\circledR} \mathrm{P}<0.01$ vs. SP600125 group. ${ }^{\text {\&\&}} \mathrm{P}<0.01$, significant differences between fold inductions.

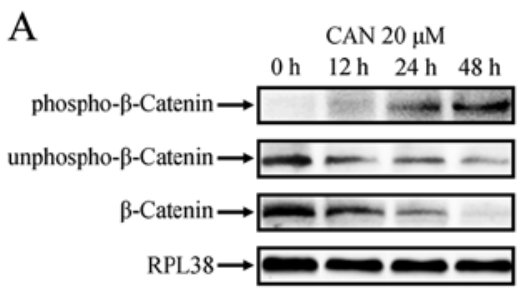

B

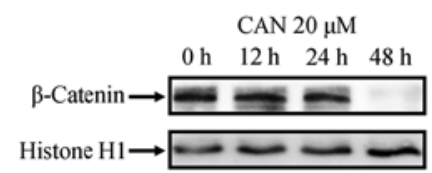

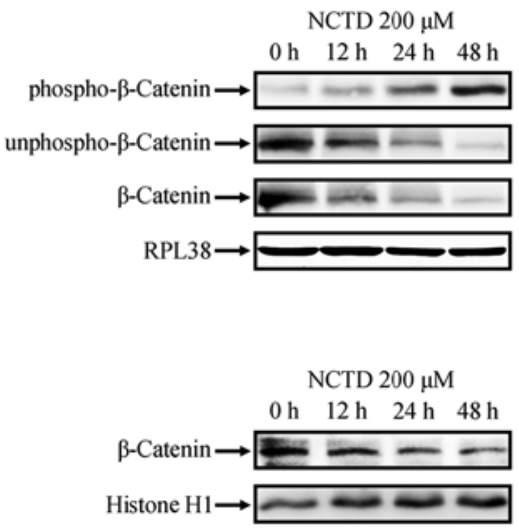
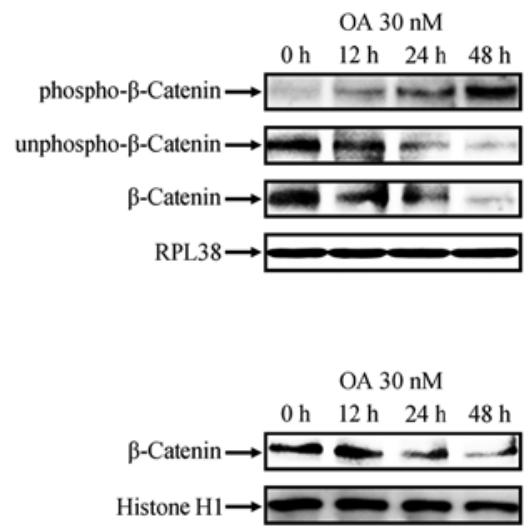

Figure 2. Treatment of PP2A inhibitors suppresses $\beta$-catenin pathway. (A) Treatment with PP2A inhibitors, CAN, NCTD and OA, increased the phosphorylation level, decreased the nonphosphorylated form (active form) of $\beta$-catenin and reduced total protein level of $\beta$-catenin. (B) Treatment with PP2A inhibitors decreased nuclear distribution of $\beta$-catenin. PP2A, protein phosphatase 2A; CAN, cantharidin; NCTD, norcantharidin; OA, okadaic acid.

We used real-time PCR to confirm the changes of these genes in the microarray analysis. Spearman's rank correlation analysis revealed that the mRNA expression values obtained by real-time PCR correlated significantly with those obtained by microarray analysis for these 13 genes $(\mathrm{P}<0.01)$. The correlation coefficient $\mathrm{R}$ ranged between 0.828 and 0.896 (Fig. 4C).

PP2A inhibitors induce expression level changes of candidate genes through $\beta$-catenin pathway-dependent manner.
To verify whether the expression changes of these 13 genes induced by PP2A inhibitors were executed through $\beta$-catenin pathway-dependent manner, the cells were pretreated with FH535, followed by treatment with PP2A inhibitors. As shown in Fig. 5, expression changes of 6 of these 13 genes, VEGFB, Dkk3, KRT8, NRP1, Cacnalg and WISP2, were attenuated by FH535 pretreatment, suggesting PP2A inhibitors downregulated these 6 genes in a $\beta$-catenin pathway-dependent manner. 

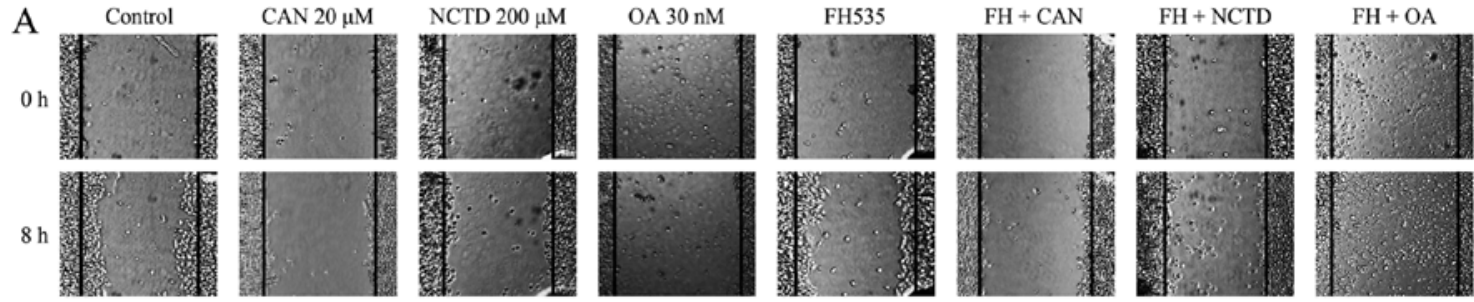

$12 \mathrm{~h}$
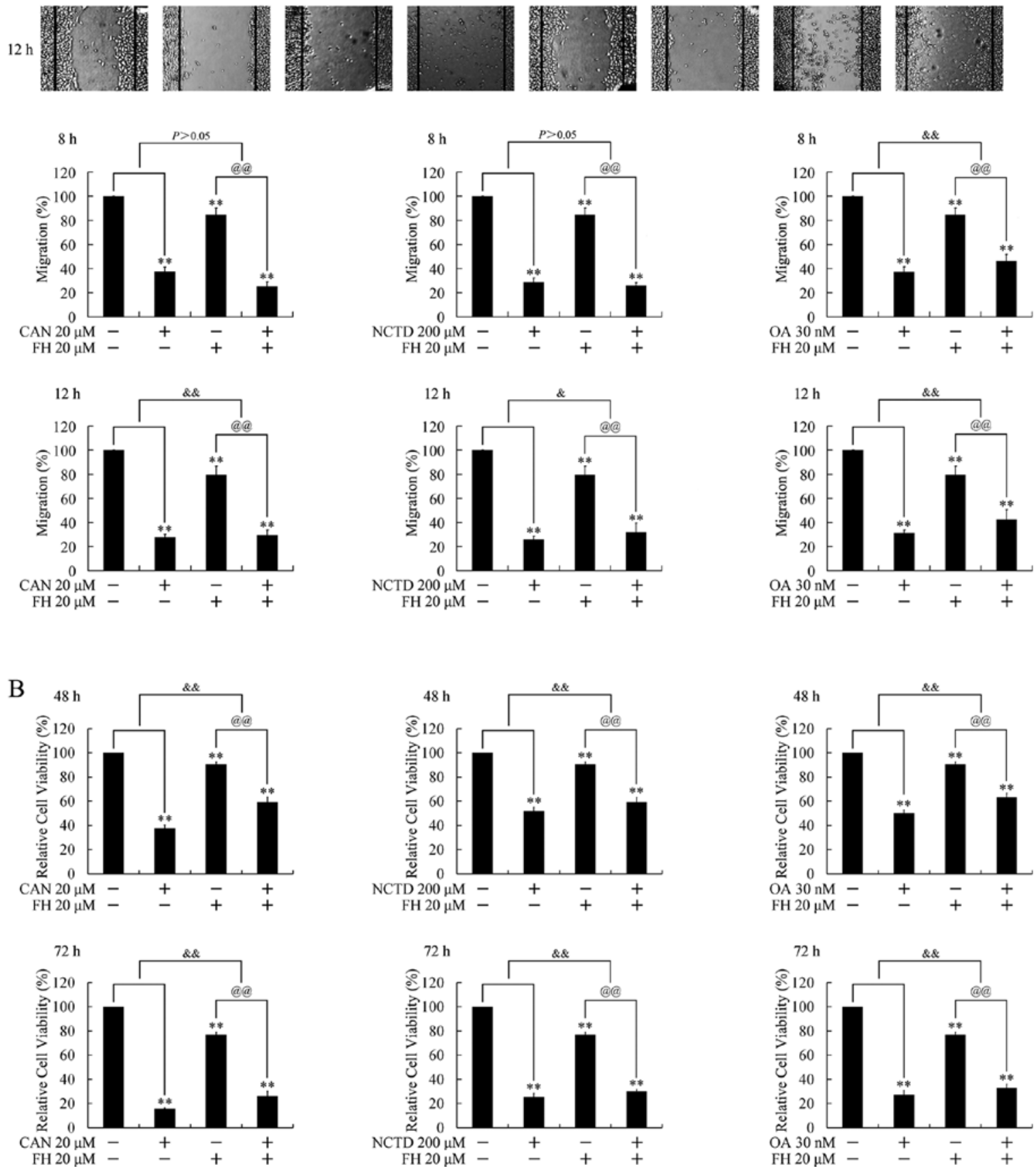

Figure 3. Pretreatment of PANC-1 cells with $\beta$-catenin pathway inhibitor attenuates the migration and growth inhibition induced by PP2A inhibitors (A) Treatment with FH535 (FH), CAN, NTCD or OA suppressed the migration of PANC-1 cells. Pretreatment with FH535 attenuated the migration inhibition induced by CAN, NTCD or OA. (B) Treatment with FH535, CAN, NTCD or OA suppressed the growth of PANC-1 cells. Pretreatment with FH535 attenuated the growth inhibition induced by $\mathrm{CAN}, \mathrm{NTCD}$ or $\mathrm{OA} .{ }^{* *} \mathrm{P}<0.01$, significant differences from the respective control groups. ${ }^{\circledR} \mathrm{P}<0.01$ vs. FH535 group. ${ }^{\&} \mathrm{P}<0.01$, significant differences between fold inductions. CAN, cantharidin; NCTD, norcantharidin; OA, okadaic acid.

\section{Discussion}

The Wnt/ $\beta$-catenin pathway has been positively confirmed to regulate cell proliferation, migration, apoptosis, differentiation and stem cell self-renewal $(16,17)$. As the indispensable mediator of classical Wnt signaling pathway, $\beta$-catenin participates in two distinct functions in the cell, depending on its localization. Membrane-localized $\beta$-catenin is isolated by the 

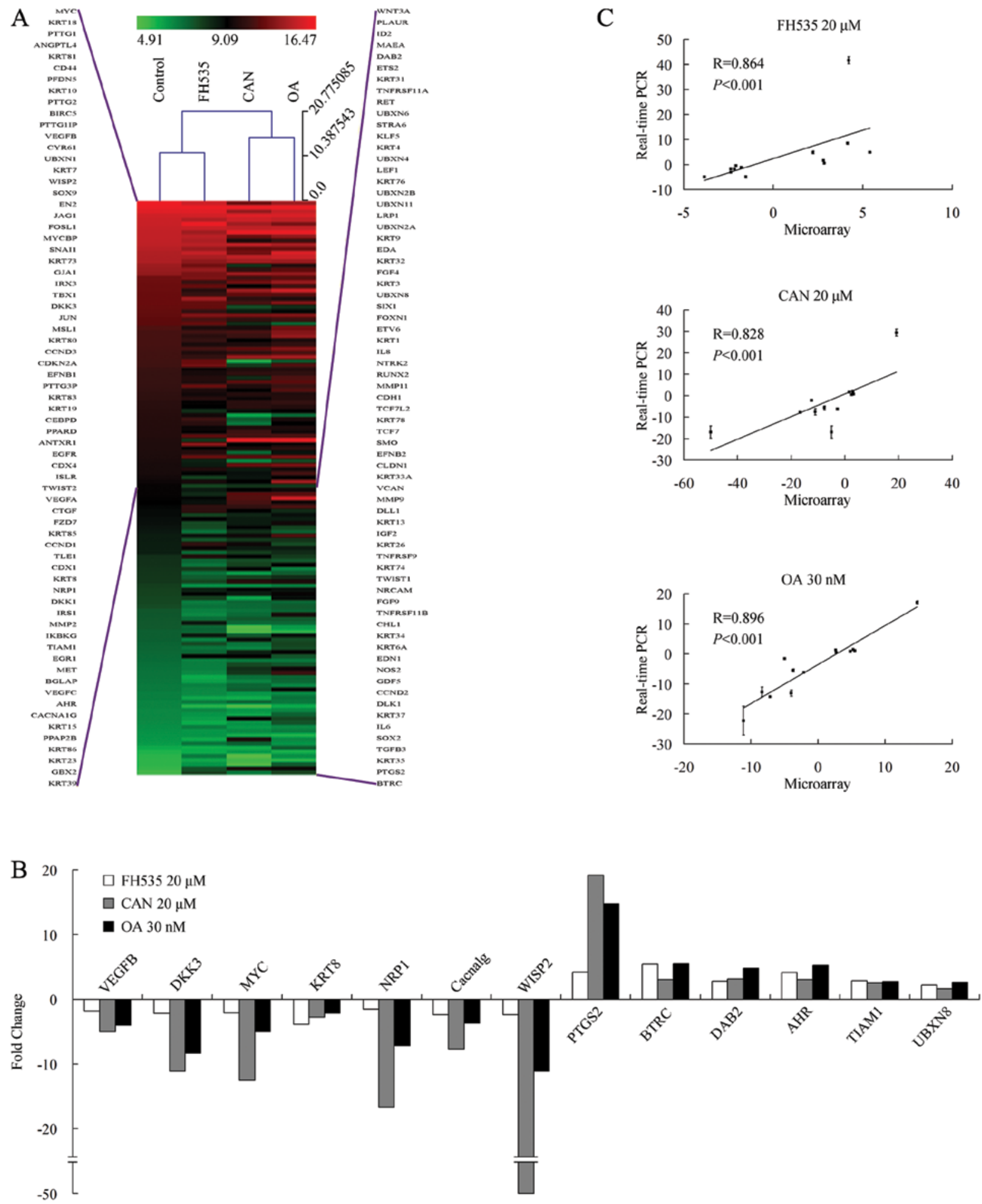

Figure 4. Regulation of expression of genes downstream of the Wnt/B-catenin pathway upon treatment with PP2A inhibitors. (A) Illustration of results of the microarrays. (B) Expression change folds of genes shared the same tendency when treated with FH535 or PP2A inhibitors. (C) Validation of the microarray results on 13 genes by real-time PCR using Spearman's rank correlation analysis. PP2A, protein phosphatase 2A.

epithelial cell-cell adhesion protein E-cadherin to chronically maintain cell-cell adhesion (9). On the other hand, classical Wnt signaling pathway causes accumulation of $\beta$-catenin in cytoplasm in complex with the transcription factor TCF/LEF that regulates target gene expression $(18,19)$. In the absence of Wnt signaling, the level of $\beta$-catenin is kept low through degradation of cytoplasmic $\beta$-catenin, which is targeted for phosphorylation and ubiquitination at Ser33/Ser37/Thr41 by GSK $3 \beta$ bound to a scaffolding complex of axin and adenomatous polyposis coli (APC) protein $(20,21)$. Thus, the key factors in $\beta$-catenin signaling are its stabilization and accumulation in the cytoplasm.

This is a reflection of an evolving literature showing $\mathrm{Wnt} / \beta$-catenin signaling has variable and sometimes para- 
A
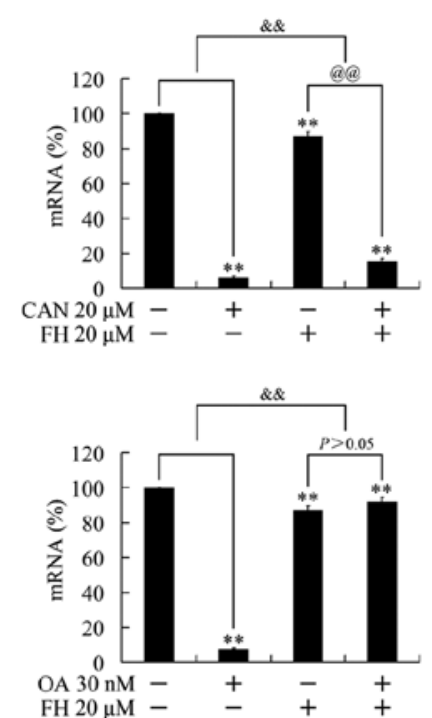

D
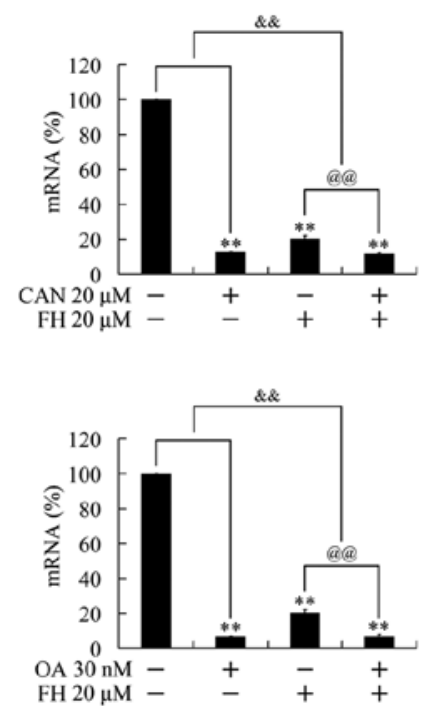

B
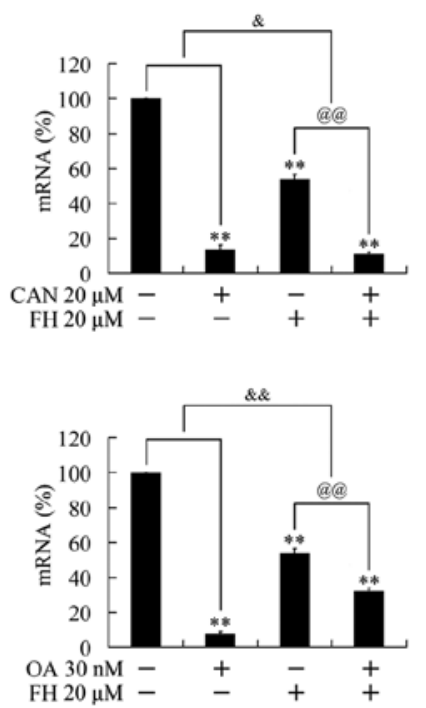

E
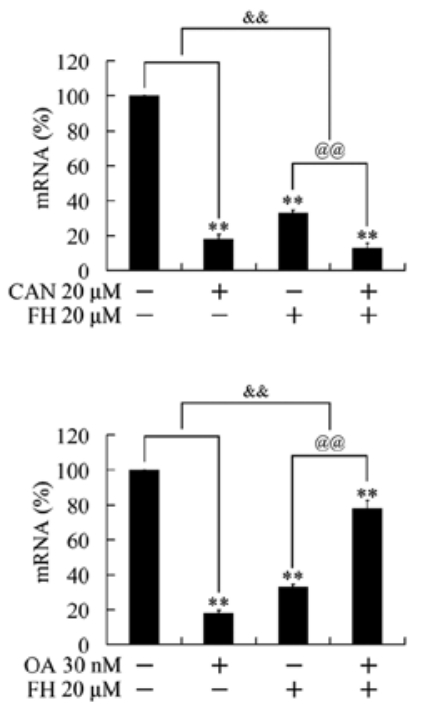

C
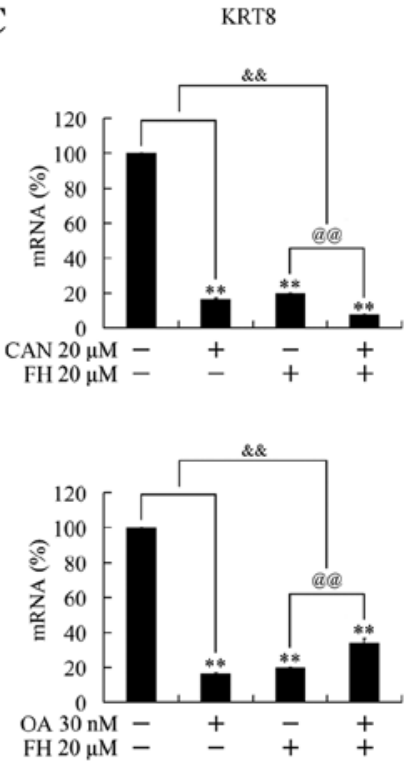

F

WISP2
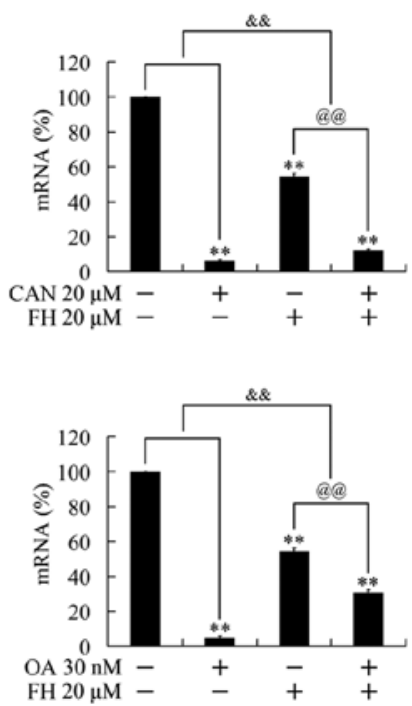

Figure 5. PP2A inhibitors induce expression changes of VEGFB, Dkk3, KRT8, NRP1, Cacnalg and WISP2 through $\beta$-catenin pathway-dependent manner in PANC-1 cells. Pretreatment of PANC-1 cells with FH535 attenuated or reversed the downregulation of VEGFB (A), Dkk3 (B), KRT8 (C), NRP1 (D), Cacnalg (E) and WISP2 (F) triggered by PP2A inhibitors. ${ }^{* *} \mathrm{P}<0.01$, significant differences from the respective control groups. ${ }^{\circledR @} \mathrm{P}<0.01$ vs. FH535 group. ${ }^{\&} \mathrm{P}<0.05$ and ${ }^{\& \&} \mathrm{P}<0.01$, significant differences between fold inductions. PP2A, protein phosphatase $2 \mathrm{~A}$.

doxical effects in the pancreas dictated by its timing, location, strength and mechanism of activation. Several investigators have demonstrated that $\beta$-catenin was essential for normal pancreatic development through the canonical Wnt signaling pathway, but this pathway is downregulated in adult pancreas (22). Although canonical activating mutations are uncommon, Wnt/ $\beta$-catenin signaling can be dysregulated in pancreatic cancer through a variety of mechanisms that modulate existing levels of autocrine or paracrine Wnt activation. There is ample in vitro and in vivo evidence that $\mathrm{Wnt} / \beta$-catenin signaling is involved in pancreatic cancer tumorigenesis. Aberration in canonical $\mathrm{Wnt} / \beta$-catenin signaling activity has been documented in pancreatic cancer (23). Positive expression of nuclear and/or cytoplasmic $\beta$-catenin is reported in anywhere from 4 to $65 \%$ of human pancreatic ductal adenocarcinoma tumors (11) and up to $40 \%$ of pancreatic intraductal papillary mucinous neoplasms (24). Positive nuclear $\beta$-catenin distribution is also reported in advanced pancreatic intraepithelial neoplasia in human and mouse model $(25,26)$. Retrospective studies reported alterations in $\beta$-catenin that correlate with tumor differentiation $(27,28)$ metastasis $(29,30)$ or patient survival $(30,31)$. Heiser et al (32) demonstrated that enhanced Wnt/ $\beta$-catenin signaling in itself could induce pancreatic tumorigenesis and activation of other oncogenes in the presence of enhanced Wnt/ $\beta$-catenin signaling induced distinct pancreatic tumor formation. This dysregulation makes it evident that these changes have meaningful phenotypic effects on pancreatic cancer tumorigenesis. The direct inhibi- 
tion of Wnt/ $\beta$-catenin signaling by knockdown of $\beta$-catenin suppresses human pancreatic cancer cell growth and survival in vitro (26). Contrary to colon cancer, in which the genetic mutations are common, the manner in which $\mathrm{Wnt} / \beta$-catenin signaling is activated and readily modulated in pancreatic cancer may also indicate that pancreatic cancer may be more amenable to genetic or pharmacological targeting of Wnt/ $\beta$-catenin as clinical therapy (11).

$\beta$-catenin is the first identified target of PP2A-B56 $\alpha$. Overexpression of $\mathrm{B} 56 \alpha$ decreased $\beta$-catenin expression in mammalian cells and Xenopus embryo explants (33). PP2A-B56 $\alpha$ is thought to have targets within Axin1-mediated degradation complex for $\beta$-catenin and it has been found to be able to inhibit the Wnt signaling pathway (34). A previous study proved that the effect of aspirin on the Wnt/ $\beta$-catenin pathway is mediated via PP2A (12). Aspirin treatment caused increased phosphorylation of Tyr307 of PP2A, an event associated with inhibition of PP2A enzymatic activity. Inhibition of PP2A resulted in phosphorylation of $\beta$-catenin and inhibition of $\beta$-catenin/TCF transcriptional activity. Although the phosphorylation-mediated degradation of $\beta$-catenin was not observed in this literature, these findings provided a molecular explanation for the efficacy of aspirin in chemoprevention of colorectal cancer and shows biochemical evidence that PP2A is an important regulator of $\mathrm{Wnt} / \beta$-catenin pathway activity in colorectal cells. In our present study, PP2A inhibitors triggered phosphorylation and degradation of $\beta$-catenin in pancreatic cancer cells, suggesting inhibition of $\beta$-catenin pathway induced by inhibition of PP2A could be a promising way in cancer treatment.

To demonstrate whether cantharidin and other PP2A inhibitors suppress pancreatic cell migration by phosphorylation/degradation of $\beta$-catenin and alter expressions of genes downstream of the Wnt/ $\beta$-catenin pathway, we firstly treated the pancreatic cancer cells with cantharidin and other PP2A inhibitors, and evaluated the migration and growth of the cells. Then, we determined the phosphorylation and protein levels of $\beta$-catenin and expression level changes of genes downstream of the Wnt/ $\beta$-catenin pathway. We found that cantharidin and other PP2A inhibitors suppressed the migration of pancreatic cells through the $\mathrm{Wnt} / \beta$-catenin pathway by phosphorylation/degradation of $\beta$-catenin. By using genome microarray technology and RT-PCR, we identified 6 candidate genes, VEGFB, NRP1, Dkk3, KRT8, Cacnalg and WISP2, at the downstream of PP2A/ $\beta$-catenin pathway.

VEGFB is a prototype member of vascular endothelial growth factor (VEGF) family, which participates in both physiologic and pathologic angiogenesis (35). VEGFB has been found to be able to promote migration and invasion, but not proliferation or survival in pancreatic cancer cells (36). NRP1 has a protumorigenic role and direct contribution to tumor progression in some studies where NRP1 is predominantly expressed in epithelial cancer cells, including carcinomas of lung, breast, prostate, pancreas and colon and is implicated in the survival, migration and invasion of tumor cells (37-40). NRPl overexpression is positively associated with metastatic potential, advanced stage, shorter 5-year survival rate and/or clinical grade in prostate (41), gastrointestinal (42) and colorectal carcinoma (43), suggesting a protumorigenic role of NRP1 and direct contribution of NRP1 to tumor progression.
Dkk3 is involved in embryonic development through its interactions with the Wnt signaling pathway. Dkk3 maintains the pancreatic cancer cells in a dedifferentiated state. Knockdown of Dkk3 resulted in significant reduction of cellular proliferation and concomitant induction of cell cycle inhibitors, as well as pancreatic epithelial cell differentiation markers (44). KRT8 (Keratin 8) is a member of the type II keratin family and knockdown of KRT8 increased migration and invasiveness, but increased apoptosis in epithelial cancer cells through upregulation of Fas receptor (45). Cacnalg $\left(\mathrm{Ca}^{2+}\right.$ channel, voltage-dependent, T-type, $\alpha 1 \mathrm{G}$ subunit) is expressed in various human tumors, including colon and pancreatic cancer and glioblastoma, as well as in acute myelogenous leukemia $(46,47)$. Knockdown of Cacnalg decreased proliferation of astrocytoma, neuroblastoma, renal tumor (48), breast cancer (49) and glioblastoma (50) cells. WISP2 (Wnt-induced secreted proteins 2), one of the three Wnt inducible proteins that belongs to the CCN family, stimulates mitosis, adhesion, apoptosis, extracellular matrix production, growth arrest and migration of multiple cell types (51). Previous findings suggest that WISP2 is relevant to tumorigenesis and malignant transformation. Its roles in cancer appear to vary depending on cell type and the microenvironment (52) and promote invasiveness and growth in the circumstance of pancreatic cancer (53). As VEGFB, NRP1, Dkk3, KRT8, Cacnalg and WISP2 all participate in the migration and/or growth regulation, the $\mathrm{PP} 2 \mathrm{~A} / \beta$-catenin pathway-mediated migration and growth inhibition induced by PP2A inhibitors could be executed through these 6 genes.

PP2A is generally considered to be a cancer suppressor. Inhibition of PP2A has been thought to be cancer promoting by induction of phosphorylation and activation of several substrate kinases, including c-Jun N-terminal kinase (JNK), extracellular signal-related kinase (ERK), p38, Akt and protein kinase $\mathrm{C}$ (PKC) amongst others, most of which can accelerate growth (54-55). However, we previously reported some kinase-dependent anticancer pathways that are induced by treatment with PP2A inhibitors, which suggested that the activation of kinase pathways may not always be cancer promoting. These kinase-dependent anticancer mechanisms induced by PP2A inhibitors include JNK-dependent growth inhibition (5), NF- $\kappa \mathrm{B}$ pathway-dependent apoptosis induction (4) and PKC-dependent downregulation of $\alpha 2$ integrin (6). In the present study, we confirmed that the phosphorylationmediated inhibition of the $\beta$-catenin pathway participated in the migration and growth inhibition effect of PP2A inhibitors.

The JNK pathway, over-activation of which suppresses the growth of pancreatic cancer cells (5), fulfilled its positive effect on migration upon treatment with PP2A inhibitors. A similar phenomenon was also found in our previous studies. The activation of PKC decreased the expression of intergrin and suppressed the adhesion to platelet, which suppress the metastasis potential. However, the activation of PKC impaired the growth inhibition effect of PP2A inhibitors. These may be ascribed to the multiple-targeting effects of PP2A, i.e., an inhibition of PP2a may cause dysfunction of abundant pathways of PP2A and the anticancer effect of PP2A inhibitors is a joint function of several pathways. These also suggest that a signaling pathway may present opposite effects in different cellular processes, which may also be affected by several signal pathways. 
In conclusion, we demonstrated that PP2A inhibitors suppressed pancreatic cancer cell migration and growth through the Wnt/ $\beta$-catenin pathway by phosphorylation and further degradation of $\beta$-catenin. This may be attributed to the selective downregulation of genes downstream of the Wnt/ $\beta$-catenin pathway. Our findings provide a promising strategy for treatment of pancreatic cancer by targeting PP2A using PP2A inhibitors.

\section{Acknowledgements}

This study was supported by grants from the National Natural Science Foundation of China (nos. 81101867, 81272542, 81200369 and 81372443), the China International Medical Foundation (CIMF-F-H001-057), the Scientific Research Project of Jiangsu Provincial Bureau of Traditional Chinese Medicine (L213236), the Medical Scientific Research Project of Jiangsu Provincial Bureau of Health (Z201206), the Special Foundation of Wu Jieping Medical Foundation for Clinical Scientific Research (nos. 320.6753.1225 and 320.6750.12242), the Science and Education for Health Foundation of Suzhou for Youth (nos. SWKQ1003 and SWKQ1011), the Scientific Research and Innovation Plan Project of Jiangsu Province for Postgraduate (CXLX13_838) and the Science and Technology Project Foundation of Suzhou (nos. SYS201112, SYSD2012137 and SYS201335).

\section{References}

1. Wolfgang CL, Herman JM, Laheru DA, et al: Recent progress in pancreatic cancer. CA Cancer J Clin 63: 318-348, 2013.

2. Paulson AS, Tran Cao HS, Tempero MA and Lowy AM: Therapeutic advances in pancreatic cancer. Gastroenterology 144 1316-1326, 2013

3. Li W, Xie L, Chen Z, et al: Cantharidin, a potent and selective PP2A inhibitor, induces an oxidative stress-independent growth inhibition of pancreatic cancer cells through G2/M cell-cycle arrest and apoptosis. Cancer Sci 101: 1226-1233, 2010.

4. Li W, Chen Z, Zong Y, et al: PP2A inhibitors induce apoptosis in pancreatic cancer cell line PANC-1 through persistent phosphorylation of IKK $\alpha$ and sustained activation of the NF- $\mathrm{NB}$ pathway. Cancer Lett 304: 117-127, 2011.

5. Li W, Chen Z, Gong FR, et al: Growth of the pancreatic cancer cell line PANC-1 is inhibited by protein phosphatase $2 \mathrm{~A}$ inhibitors through overactivation of the c-Jun N-terminal kinase pathway. Eur J Cancer 47: 2654-2664, 2011.

6. Shou LM, Zhang QY, Li W, et al: Cantharidin and norcantharidin inhibit the ability of MCF-7 cells to adhere to platelets via protein kinase $\mathrm{C}$ pathway-dependent downregulation of $\alpha 2$ integrin. Oncol Rep 30: 1059-1066, 2013.

7. Clevers $\mathrm{H}$ and Nusse R: Wnt/beta-catenin signaling and disease. Cell 149: 1192-1205, 2012.

8. MacDonald BT, Tamai K and He X: Wnt/beta-catenin signaling: components, mechanisms, and diseases. Dev Cell 17: 9-26, 2009.

9. Nelson WJ and Nusse R: Convergence of Wnt, beta-catenin, and cadherin pathways. Science 303: 1483-1487, 2004.

10. Fearon ER: PARsing the phrase 'all in for Axin'- Wnt pathway targets in cancer. Cancer Cell 16: 366-368, 2009.

11. White BD, Chien AJ and Dawson DW: Dysregulation of $\mathrm{Wnt} / \beta$-catenin signaling in gastrointestinal cancers. Gastroenterology 142: 219-232, 2012.

12. Bos CL, Kodach LL, van den Brink GR, et al: Effect of aspirin on the Wnt $/ \beta$-catenin pathway is mediated via protein phosphatase 2A. Oncogene 25: 6447-6456, 2006.

13. Carmichael J, DeGraff WG, Gazdar AF, Minna JD and Mitchell JB: Evaluation of a tetrazolium-based semiautomated colorimetric assay: assessment of chemosensitivity testing. Cancer Res 47: 936-942, 1987.

14. Huang C, Jacobson K and Schaller MD: MAP kinases and cell migration. J Cell Sci 117: 4619-4628, 2004.
15. Handeli S and Simon JA: A small-molecule inhibitor of Tcf/beta-catenin signaling down-regulates PPARgamma and PPARdelta activities. Mol Cancer Ther 7: 521-529, 2008.

16. Turashvili G, Bouchal J, Burkadze G and Kolar Z: Wnt signaling pathway in mammary gland development and carcinogenesis. Pathobiology 73: 213-223, 2006.

17. Yamaguchi TP: Heads or tails: Wnts and anterior-posterior patterning. Curr Biol 11: R713-R724, 2001.

18. Tetsu $\mathrm{O}$ and McCormick F: Beta-catenin regulates expression of cyclin D1 in colon carcinoma cells. Nature 398: 422-426, 1999.

19. Clevers H: Wnt/beta-catenin signaling in development and disease. Cell 127: 469-480, 2006.

20. Polakis P: Wnt signaling and cancer. Genes Dev 14: 1837-1851, 2000.

21. Takahashi-Yanaga $\mathrm{F}$ and Sasaguri T: GSK-3beta regulates cyclin D1 expression: a new target for chemotherapy. Cell Signal 20: 581-589, 2008.

22. Papadopoulou S and Edlund $\mathrm{H}$ : Attenuated Wnt signaling perturbs pancreatic growth but not pancreatic function. Diabetes 54: 2844-2851, 2005.

23. Zeng G, Germinaro M, Micsenyi A, et al: Aberrant Wnt/betacatenin signaling in pancreatic adenocarcinoma. Neoplasia 8: 279-289, 2006

24. Chetty R, Serra S, Salahshor S, et al: Expression of Wnt-signaling pathway proteins in intraductal papillary mucinous neoplasms of the pancreas: a tissue microarray analysis. Hum Pathol 37: 212-217, 2006.

25. Pasca di Magliano M, Biankin AV, Heiser PW, et al: Common activation of canonical Wnt signaling in pancreatic adenocarcinoma. PloS One 2: e1155, 2007.

26. Wang L, Heidt DG, Lee CJ, et al: Oncogenic function of ATDC in pancreatic cancer through Wnt pathway activation and beta-catenin stabilization. Cancer Cell 15: 207-219, 2009.

27. Lowy AM, Fenoglio-Preiser C, Kim OJ, et al: Dysregulation of beta-catenin expression correlates with tumor differentiation in pancreatic duct adenocarcinoma. Ann Surg Oncol 10: 284-290, 2003.

28. Joo YE, Rew JS, Park CS and Kim SJ: Expression of E-cadherin, alpha- and beta-catenins in patients with pancreatic adenocarcinoma. Pancreatology 2: 129-137, 2002.

29. Karayiannakis AJ, Syrigos KN,Polychronidis A and Simopoulos C: Expression patterns of alpha-, beta- and gamma-catenin in pancreatic cancer: correlation with E-cadherin expression, pathological features and prognosis. Anticancer Res 21: 4127-4134, 2001.

30. Li YJ, Wei ZM, Meng YX and Ji XR: Beta-catenin up-regulates the expression of cyclinD1, c-myc and MMP-7 in human pancreatic cancer: relationships with carcinogenesis and metastasis. World J Gastroenterol 11: 2117-2123, 2005.

31. Qiao Q, Ramadani M, Gansauge S, Gansauge F, Leder G and Beger HG: Reduced membranous and ectopic cytoplasmic expression of beta -catenin correlate with cyclin D1 overexpression and poor prognosis in pancreatic cancer. Int J Cancer 95: 194-197, 2001.

32. Heiser PW, Cano DA, Landsman L, et al: Stabilization of beta-catenin induces pancreas tumor formation. Gastroenterology 135: 1288-1300, 2008.

33. Seeling JM, Miller JR, Gil R, Moon RT, White R and Virshup DM: Regulation of beta-catenin signaling by the B56 subunit of protein phosphatase 2A. Science 283: 2089-2091, 1999.

34. Li X, Yost HJ, Virshup DM and Seeling JM: Protein phosphatase 2A and its B56 regulatory subunit inhibit Wnt signaling in Xenopus. EMBO J 20: 4122-4131, 2001.

35. Ferrara N, Gerber HP and LeCouter J: The biology of VEGF and its receptors. Nat Med 9: 669-676, 2003.

36. Wey JS, Fan F, Gray MJ, et al: Vascular endothelial growth factor receptor-1 promotes migration and invasion in pancreatic carcinoma cell lines. Cancer 104: 427-438, 2005.

37. Bielenberg DR, Pettaway CA, Takashima S and Klagsbrun M: Neuropilins in neoplasms: expression, regulation, and function. Exp Cell Res 312: 584-593, 2006.

38. Frankel P, Pellet-Many C, Lehtolainen P, et al: Chondroitin sulphate-modified neuropilin 1 is expressed in human tumour cells and modulates 3D invasion in the U87MG human glioblastoma cell line through a p130Cas-mediated pathway. EMBO Rep 9: 983-989, 2008

39. Miao HQ, Lee P, Lin H, Soker S and Klagsbrun M: Neuropilin-1 expression by tumor cells promotes tumor angiogenesis and progression. FASEB J 14: 2532-2539, 2000. 
40. Wey JS, Gray MJ, Fan F, et al: Overexpression of neuropilin-1 promotes constitutive MAPK signalling and chemoresistance in pancreatic cancer cells. Br J Cancer 93: 233-241, 2005.

41. Latil A, Bieche I, Pesche S, et al: VEGF overexpression in clinically localized prostate tumors and neuropilin-1 overexpression in metastatic forms. Int J Cancer 89: 167-171, 2000.

42. Hansel DE, Wilentz RE, Yeo CJ, Schulick RD, Montgomery E and Maitra A: Expression of neuropilin-1 in high-grade dysplasia invasive cancer, and metastases of the human gastrointestinal tract. Am J Surg Pathol 28: 347-356, 2004.

43. Ochiumi T, Kitadai Y, Tanaka S, Akagi M, Yoshihara M and Chayama K: Neuropilin-1 is involved in regulation of apoptosis and migration of human colon cancer. Int J Oncol 29: 105-116, 2006.

44. Zenzmaier C, Hermann M, Hengster P and Berger P: Dickkopf-3 maintains the PANC-1 human pancreatic tumor cells in a dedifferentiated state. Int J Oncol 40: 40-46, 2012.

45. Fortier AM, Asselin E and Cadrin M: Keratin 8 and 18 loss in epithelial cancer cells increases collective cell migration and cisplatin sensitivity through claudin 1 up-regulation. J Biol Chem 288: 11555-11571, 2013.

46. Toyota M, Ho C, Ohe-Toyota M, Baylin SB and Issa JP: Inactivation of CACNA1G, a T-type calcium channel gene, by aberrant methylation of its 5' $\mathrm{CpG}$ island in human tumors. Cancer Res 59: 4535-4541, 1999.

47. Lory P, Bidaud I and Chemin J: T-type calcium channels in differentiation and proliferation. Cell Calcium 40: 135-146, 2006.
48. Panner A and Wurster RD: T-type calcium channels and tumor proliferation. Cell calcium 40: 253-259, 2006.

49. Taylor JT, Huang L, Pottle JE, et al: Selective blockade of T-type $\mathrm{Ca}^{2+}$ channels suppresses human breast cancer cell proliferation. Cancer Lett 267: 116-124, 2008.

50. Zhang Y, Zhang J, Jiang D, et al: Inhibition of T-type $\mathrm{Ca}^{2+}$ channels by endostatin attenuates human glioblastoma cell proliferation and migration. Br J Pharmacol 166: 1247-1260, 2012.

51. Brigstock DR: The CCN family: a new stimulus package. J Endocrinol 178: 169-175, 2003.

52. Davies SR, Davies ML, Sanders A, Parr C, Torkington J and Jiang WG: Differential expression of the CCN family member WISP-1, WISP-2 and WISP-3 in human colorectal cancer and the prognostic implications. Int J Oncol 36: 1129-1136, 2010.

53. Aikawa T, Gunn J, Spong SM, Klaus SJ and Korc M: Connective tissue growth factor-specific antibody attenuates tumor growth, metastasis, and angiogenesis in an orthotopic mouse model of pancreatic cancer. Mol Cancer Ther 5: 1108-1116, 2006.

54. Millward TA, Zolnierowicz S and Hemmings BA: Regulation of protein kinase cascades by protein phosphatase 2A. Trends Biochem Sci 24: 186-191, 1999.

55. Janssens V, Goris J and Van Hoof C: PP2A: the expected tumor suppressor. Curr Opin Genet Dev 15: 34-41, 2005. 